\title{
A Study of Clinicopathological Correlation in Patients with Lupus Nephritis in a Tertiary Centre in South India
}

\author{
Abhijith S. Magal ${ }^{1}$, Vasudeva Acharya ${ }^{2}$, Ravindra Prabhu A. ${ }^{3}$ \\ ${ }^{1,2}$ Department of Medicine, Kasturba Medical College, Manipal, Karnataka, India. \\ ${ }^{3}$ Department of Nephrology, Kasturba Medical College, Manipal, Karnataka, India.
}

\section{ABSTRACT}

\section{BACKGROUND}

Lupus nephritis (LN) is very common and significantly affects the prognosis of patients with systemic lupus erythematosus (SLE). Although the diagnosis of LN is obvious sometimes from the simple lab tests, it is important to perform a renal biopsy. We wanted to study the correlation between the clinical, lab parameters and the histopathological grade of LN in a tertiary care centre in South India.

\section{METHODS}

A cross-sectional study was conducted among all patients with biopsy proven LN who underwent a renal biopsy from 19 / 09 / 2017 to 30 / 06 / 2019. Data of 51 patients were obtained. For all the study subjects, a history was obtained, clinical examination was done, and their lab parameters and the renal biopsy report were noted in the proforma. The histology was correlated with the laboratory and clinical data.

\section{RESULTS}

The majority of the patients were females. Maximum patients had class IV LN and class III had more males than any other class of LN. When clinical and laboratory parameters were correlated with histopathological findings, pedal oedema and facial puffiness were found to be correlated with the classes of lupus nephritis with statistical significance. Among investigations, haemoglobin, C3, activity score and 24 -hour urine protein had a statistically significant correlation with the various classes of lupus nephritis.

\section{CONCLUSIONS}

This study suggests some meaningful correlation between pedal oedema, facial puffiness, 24 hours urine protein, haemoglobin levels, C3 levels and activity score with lupus nephritis classes of ISN / RPS 2003 classification.

\section{KEY WORDS}

Lupus, Clinicopathological Correlation
Corresponding Author: Dr. Abhijith S. Magal, Department of Medicine, Kasturba Medical College, Manipal, Udupi - 576104, Karnataka, India. E-mail:abhijith.magal01@gmail.com

DOI: $10.14260 /$ jemds/2021/569

How to Cite This Article: Magal AS, Acharya V, Prabhu AR. A study of clinicopathological correlation in patients with lupus nephritis in a tertiary centre in South India. J Evolution Med Dent Sci 2021;10(33):2789-2794, DOI: $10.14260 /$ jemds/2021/569

Submission 15-05-2021,

Peer Review 09-07-2021,

Acceptance 15-07-2021,

Published 16-08-2021.

Copyright (c) 2021 Abhijith S. Magal et al. This is an open access article distributed under Creative Commons Attribution License [Attribution 4.0 International (CC BY 4.0)] 


\section{BACKGROUND}

Systemic lupus erythematosus (SLE) is a chronic autoimmune disease which affects almost every organ system of the body, but very often involves the kidney ${ }^{1}$ It is especially common among young women with the age of onset between 15 - 40 and a female to male ratio of 9:11 Although multiple studies have estimated the incidence and prevalence of SLE in western countries, there is still a paucity of information regarding the incidence and prevalence of it in India. One such study done in 1993 gave a point prevalence of SLE of 3.2 per 100,000 in the Indian population. ${ }^{2}$

Renal involvement in SLE, also called lupus nephritis (LN) is so common that at least $50 \%$ of patients with SLE exhibit some features of renal involvement at some time during the disease course. ${ }^{3}$ LN is said to affect the course of SLE in a patient and is also a major predictor of morbidity and mortality in the SLE population. 4,5 The natural history of LN is also highly heterogeneous, varying from subclinical proteinuria to aggressive glomerulonephritis that may progress to end-stage renal disease (ESRD).5,6 Although the diagnosis of LN is obvious in an SLE patient presenting with proteinuria, abnormal renal function and active urinary sediment, it is important to perform a renal biopsy for the following reasons - first, abnormal renal pathology can also occur without the above mentioned features. ${ }^{7}$ Second, it is pertinent for accurate determination of severity and hence the prognosis and lastly, it is an important guide to therapy in LN patients. The renal biopsy specimens are first evaluated by light microscopy, immunofluorescence and electron microscopy and are then categorized according to different classification systems. The International Society of Nephrology / Renal Pathology Society (ISN / RPS) 2003 classification of LN is the most commonly used system today. ${ }^{8}$ The ISN / RPS 2003 includes six principal pathological patterns (Classes I-VI).

The nature and severity of the clinical features and the lab investigations in a patient with LN cannot accurately predict the underlying histological severity. Nonetheless, it is generally agreed upon that certain lab and clinical findings correlate with the renal histological features. Although there have been multiple studies addressing the clinico pathological correlation in western countries, ${ }^{9-12}$ there are very few studies done in India.13,14 This is even more pertinent in a developing nation like India because of the lack of access to novel biomarkers and lack of adequate resources. Our objective was to study the correlation between the clinical, lab parameters and the histopathological grade of LN in a tertiary care centre in South India.

\section{METHODS}

A cross-sectional observational study was conducted for all patients with biopsy proven LN. Patients with LN who underwent a renal biopsy during the study period from 19 / 09 / 2017 to 30 / 06 / 2019 in Kasturba Hospital, Manipal were included in the study. The renal biopsy specimens were sent to a nephropathologist in Bangalore, India and LN cases were classified according to the 2003 ISN / RPS classification. ${ }^{8}$ Data regarding immunofluorescence findings, activity indices (AIs) and chronicity indices (CIs) and any other additional findings were also available in the biopsy report. Sample size calculated based on previous studies, hospital data and based on an $80 \%$ anticipated agreement with $20 \%$ relative error was 39 . Consequently, data of 51 patients were obtained.

The research study was approved by the Kasturba hospital institutional ethical review board. Approval number is IEC 610 / 2017. For all the study subjects, after a written informed consent, a history was obtained, and the clinical examination was done and their lab parameters were noted in the proforma. History from the patient included demographic details (sex, age), history of haematuria, decreased urine output, pedal oedema, facial puffiness, rash, arthralgia, Raynaud's phenomenon, and a family history of SLE. The patients were then examined and the vitals including pulse, blood pressure were noted, a general examination was done and the presence of pallor, rash, oedema were noted. A systemic examination was also done including the examination of joints. The laboratory values that were noted for each subject were haemoglobin, total counts, platelets, erythrocyte sedimentation rate (ESR), urine protein and microscopy, urea, creatinine and electrolytes, C reactive protein (CRP), Anti-nuclear antibody (ANA) global and profile, anti - dsDNA (double - stranded DNA) titres, 24 hour urinary protein, complements - C3, C4 and any other auto - antibodies including anti-cardiolipin antibody, lupus anticoagulant. Finally, the renal biopsy report was noted including ISN / RPS stage, activity and chronicity index and any other additional features if present.

\section{Statistical Analysis}

All statistical evaluations were performed using SPSS version 25.0 (SPSS, Chicago, IL, USA). Data were summarized as mean $\pm \mathrm{SD}$, median and inter - quartile range. Pearson's correlation and chi-square tests were used to compare frequency variables and correlation among different variables. Univariate analysis of variance (ANOVA) with post hoc analysis by Dunnett $t$ was used for the association between continuous variables and the Kruskal - Wallis test was used to test the association between non normally distributed continuous variables. A $P$ value of $<0.05$ was considered statistically significant.

\section{RESULTS}

A total of 51 patients who were biopsy - proven cases of lupus nephritis was enrolled in the study. Their mean age was $28.25 \pm 10.50$ years. Out of the 51 patients, 6 were males constituting $12 \%$ and 45 were females constituting $88 \%$ of the total patients enrolled. Among all the patients enrolled in the study, $20 \%(\mathrm{~N}=6)$ had class II LN, $25 \%(\mathrm{~N}=13)$ had class III LN, $43 \%(\mathrm{~N}=22)$ had class IV LN and $20 \%(\mathrm{~N}=10)$ had class $\mathrm{V}$ LN. The analysis for gender distribution among various classes of LN revealed that class III had more males than any other class of LN.

The most common symptom cited by patients with lupus nephritis was pedal oedema which was seen in $69.2 \%(\mathrm{~N}=$ 
36) of patients followed by facial puffiness which was seen in $61.5 \%(\mathrm{~N}=32)$ of patients. The other symptoms according to their frequency of occurrence were arthralgia seen in $50 \%$ $(\mathrm{N}=26)$ of patients, rash (any form of rash including malar rash) seen in $44.2 \%(\mathrm{~N}=23)$ and decreased urine output which was seen in $17.30 \%(\mathrm{~N}=9)$. When the patients were examined, the frequency of various signs elicited in patients of LN was matching the frequency of various symptoms cited by the patients.

The investigations of patients were recorded, and it revealed the following - $13.7 \%(\mathrm{~N}=7)$ had a high creatinine value defined by an arbitrary creatinine value of $1.2 \mathrm{mg} / \mathrm{dL}$ or higher. However, when the eGFR was calculated using the CKD - EPI formula and the patients were classified as having renal failure according to the KDIGO criteria as those with GFR $<60 \mathrm{ml} / \mathrm{min} / 1.7 \mathrm{~m}^{2}$, we found that $17.6 \%(\mathrm{~N}=9)$ had renal failure. When the patient's urine was analysed, we found that $68.6 \%(\mathrm{~N}=35)$ were found to have microscopic haematuria defined as > $5 \mathrm{RBC} /$ high power field. When 24 hour urine protein quantification was done, we found that $23.5 \%(\mathrm{~N}=12)$ had nephrotic range proteinuria defined as > $3 \mathrm{~g} /$ day of proteinuria. The complements level done revealed that $82.3 \%(\mathrm{~N}=42)$ had low $\mathrm{C} 3$ levels defined as < $90 \mathrm{mg} / \mathrm{dL}$ and $76.4 \%(\mathrm{~N}=39)$ had low $\mathrm{C} 4$ levels defined as $<10 \mathrm{mg} / \mathrm{dL}$. The auto - antibodies testing revealed that 64.7 $\%(\mathrm{~N}=33)$ had ANA strong positive and $31.6 \%(\mathrm{~N}=16)$ had an anti - dsDNA titre $>800 \mathrm{IU} / \mathrm{ml}$.

When symptoms and signs of lupus nephritis were correlated with various classes of lupus nephritis, the findings found are summarized in Table 1. Patients complaining of malar rash were uniformly distributed among the various classes of lupus nephritis. Pedal oedema was found on examination in $16.7 \%$ of those with class II, $61.5 \%$ of those with class III, $77.3 \%$ of those in class IV and $80 \%$ of those in class V. Thus, pedal oedema was seen to increase across the classes with maximum seen in patients with class $\mathrm{V}$ lupus nephritis. This was found to be statistically significant. Facial puffiness was found on examination in 33.3 $\%$ of those with class II, $38.5 \%$ of those with class III, $81.8 \%$ of those in class IV and $40 \%$ of those in class V. Hence, facial puffiness was found in most patients with class IV LN and this was also statistically significant.

When the patient's investigations were correlated with the various classes of lupus nephritis, the findings found are summarized in Table 2. Haemoglobin had a statistically significant correlation with the various classes of lupus nephritis with the lowest haemoglobin recorded in class IV. When renal functions were correlated across the various classes of lupus nephritis, it was found that the maximum urea values were found in class IV (median of 40.5 with an interquartile range of $26.75,53.75$ ) and this was statistically significant. However, when creatinine was correlated with various classes, it was found that there was no significant correlation between the various classes. Creatinine was distributed uniformly across the various classes. When eGFR was calculated using the CKD - EPI formula and was correlated across the classes of lupus nephritis, it was found that eGFR gradually decreased as the class increased. However, this correlation did not show statistical significance. The number of urine RBCs per high power field was found to increase across the classes and patients in class $\mathrm{V}$ lupus nephritis had maximum RBCs in the urine. This correlation, however, wasn't found to be statistically significant. 24-hour urine protein was found to increase across the classes and maximum patients in class $\mathrm{V}$ lupus nephritis had nephrotic range proteinuria (Figure 1). This correlation was found to be statistically significant.

When the complement levels were correlated with the various classes of lupus nephritis, it was found that C3 levels correlated significantly with the various classes with the lowest C3 levels noted in class IV (Figure 2). However, C4 levels did not show any statistically significant correlation.

The activity score and the chronicity score obtained from the renal biopsy report were correlated with the various classes of lupus nephritis and it was found that the activity scores correlated significantly with the various classes. The activity score was found to be the highest in class IV with a median of 7.5. However, the chronicity score did not correlate significantly with the various classes.

\begin{tabular}{|c|c|c|c|c|c|}
\hline Symptoms and Signs & Class II $(\mathrm{N}=6)$ & Class III $(\mathrm{N}=13)$ & Class IV $(\mathrm{N}=22)$ & Class V $(\mathrm{N}=10)$ & P Value \\
\hline Haematuria & $0 / 6(0 \%)$ & $0 / 13(0 \%)$ & $1 / 22(4.5 \%)$ & $0 / 10(0 \%)$ & 0.719 \\
\hline Malar rash & $3 / 6(50 \%)$ & $4 / 13(30.8 \%)$ & $4 / 22(18.2 \%)$ & $4 / 10(40 \%)$ & 0.375 \\
\hline Decreased urine output & $2 / 6(33.3 \%)$ & $0 / 13(0 \%)$ & $6 / 22(27.3 \%)$ & $1 / 10(10 \%)$ & 0.132 \\
\hline Arthralgia & $4 / 6(66.7 \%)$ & $7 / 13(53.8 \%)$ & $12 / 22(54.5 \%)$ & $3 / 10(30 \%)$ & 0.474 \\
\hline Pedal oedema & $1 / 6(16.7 \%)$ & $8 / 13(61.5 \%)$ & 17 / $22(77.3 \%)$ & $8 / 10(80 \%)$ & 0.032 \\
\hline Facial Puffiness & $2 / 6(33.3 \%)$ & $5 / 13(38.5 \%)$ & $18 / 22(81.8 \%)$ & $4 / 10(40 \%)$ & 0.019 \\
\hline
\end{tabular}

\begin{tabular}{|c|c|c|c|c|c|}
\hline Investigations & Class II $(\mathrm{N}=6)$ & Class III ( $\mathrm{N}=13)$ & Class IV $(\mathrm{N}=22)$ & Class V $(\mathrm{N}=10)$ & P Value \\
\hline Haemoglobin (g / dL) & $11.00 \pm 1.86$ & $11.33 \pm 1.15$ & $9.74 \pm 1.73$ & $11.36 \pm 1.88$ & 0.019 \\
\hline $\operatorname{TLC}(/ \mu \mathrm{l})$ & $5500(3175,11550)$ & $8700(5900,9700)$ & $6200(4275,7175)$ & $7900(4950,11200)$ & 0.082 \\
\hline Urea (mg / dL) & $26.5(14,30.75)$ & $23(13.5,39)$ & $40.5(26.75,53.75)$ & $26.5(14.5,42)$ & 0.021 \\
\hline Creatinine $(\mathrm{mg} / \mathrm{dL})$ & $0.55(0.47,0.97)$ & $0.8(0.6,1.25)$ & $0.75(0.6,1.2)$ & $0.75(0.5,1.02)$ & 0.949 \\
\hline eGFR $\left(\mathrm{ml} / \mathrm{min} / 1.73 \mathrm{~m}^{2}\right)$ & $117.7 \pm 33.2$ & $101.4 \pm 43.1$ & $100.7 \pm 38.4$ & $98.5 \pm 42.2$ & 0.796 \\
\hline Urinary RBCs & $3(0,14)$ & $2(1,4)$ & $8(2,20)$ & $11(6,19)$ & 0.108 \\
\hline $24 \mathrm{hr}$ urinary protein $(\mathrm{mg} / 24 \mathrm{hr}$ ) & $356(231.75,2331.75)$ & $726(393,1899)$ & $2130.5(566.25,3103)$ & $3151(1093.5,5640)$ & 0.026 \\
\hline C3 (mg / dL) & $49(32,81.75)$ & $56(31,79)$ & $25(14,52)$ & $48.5(28.25,85.5)$ & 0.014 \\
\hline C4 (mg / dL) & $6(2.5,21)$ & $4(2.5,11.5)$ & $4.5(3,10.5)$ & $6.5(4.75,18.5)$ & 0.258 \\
\hline Activity Score & $0(0,0)$ & $2(1,4)$ & $7.5(6,9.25)$ & $2(1,6.5)$ & $<0.001$ \\
\hline ANA Strong positive & 2 / 6 (33.3\%) & $8 / 13(61.5 \%)$ & $16 / 22(72.7 \%)$ & $7 / 10(70 \%)$ & 0.336 \\
\hline Anti-ds - DNA >800 (IU / ml $)$ & $1 / 6(16.6 \%)$ & $5 / 13(38.4 \%)$ & $9 / 22(40.9 \%)$ & $3 / 10(30 \%)$ & 0.707 \\
\hline Anti-ds - DNA titres (IU / ml) & $585 \pm 202.73$ & $628 \pm 262.11$ & $717.35 \pm 113.74$ & $588.57 \pm 257.12$ & 0.474 \\
\hline \multicolumn{6}{|c|}{ Table 2. Correlation of Investigations with the Biopsy Finding } \\
\hline \multicolumn{6}{|c|}{$\begin{array}{l}\text { Categorical variables compared across various classes of LN are expressed as frequencies in numbers (\%) } \\
\text { Normally distributed numerical variables compared across various classes of LN are expressed in mean } \pm \text { SD } \\
\text { Non - normally distributed numerical variables compared across various classes of LN are expressed as Median (interquartile range) }\end{array}$} \\
\hline
\end{tabular}


The autoantibodies in lupus nephritis were correlated across the various classes of lupus nephritis. Initially, when the results of ANA global were compared across classes, it was found that maximum patients in class IV had ANA strong positive $(72.7 \%)$. When the number of patients with antidsDNA titre $>800 \mathrm{IU} / \mathrm{ml}$ was compared across the classes, it was found that 9 out of 22 patients (40.9\%) in class IV had anti - dsDNA titres of $>800 \mathrm{IU} / \mathrm{ml}$. However, both of these did not correlate significantly with the various classes of LN. When the actual anti - dsDNA titres were correlated with the various classes, it was found that class IV had the maximum titres with a mean of $717.35 \mathrm{IU} / \mathrm{ml}$. However, this correlation, too, was not found to be statistically significant.

\section{DISCUSSION}

In our study, we tried to see the correlation between clinical features, laboratory parameters and histopathological features including the activity and chronicity indices. Our study found the mean age of those with LN as 28.25 years with a female to male ratio of 7.5:1. The female preponderance and the mean age found in this study were similar to various other studies published. ${ }^{11,12,14}$ This is owing to the fact that SLE is mainly a disease of females of childbearing age. The reason for this, even though uncertain, is attributed to differences in the metabolism of sex hormones and / or gonadotropin - releasing hormones.

In this study, $20 \%$ of patients had class II LN, $25 \%$ had class III LN and $20 \%$ had class V LN. However, the vast majority of patients presented with Class IV LN ( $43 \%$ of the total patients sampled had class IV LN), similar to that seen in other studies. Also, patients with class IV LN were more commonly associated with the presence of symptoms like facial puffiness, pedal oedema and this was found to be statistically significant in our study. They also had increased proteinuria and high dsDNA titres. This, too, is consistent with other studies published.11-13,15 This is extremely important because up to $40 \%$ of the patients with class IV LN die or lose their renal function within 5 years after diagnosis. ${ }^{12}$ Due to its relative severity, class IV LN is the most important form of renal disease complicating SLE. 16 This form of glomerular disease has a widely variable course and this requires clinical and pathological factors that would enable better prognostication and management of the disease. Studies have also consistently shown that the majority of patients with Class IV LN had hypertension 17,18 and this was associated with a poor outcome. ${ }^{19,20}$ This, however, was not the case in our study where we found that there was not much of a difference in the systolic blood pressure among the various classes of LN.

We found that haemoglobin values were significantly correlated among the various classes of LN with the lowest haemoglobin value recorded in patients with class IV LN. This was also reported by two other studies, one of which was done here in Karnataka, India by Shobha V PR et al. ${ }^{21,22}$ The probable explanations proposed by the above studies for the same are that those with class IV LN have a higher risk of developing anaemia secondary to renal failure and they also have maximum haematuria. However, our study did not find significant haematuria in those with class IV LN. Also, in our study, Class IV LN had a mean eGFR of $100.7 \mathrm{ml} / \mathrm{min}$ / $1.73 \mathrm{~m}^{2}$. Hence, with the data at hand, we cannot attribute the anaemia to renal failure.

When the renal function was compared across the classes of LN, we found that even though eGFR decreased with an increasing class of $\mathrm{LN}$, it did not reach statistical significance. We failed to find a correlation between creatinine and the classes of LN, even though various studies have shown that an elevated serum creatinine at the time of renal biopsy is a strong adverse prognostic marker.12,13,19,23 We, however, found that serum urea levels were significantly correlated with the various classes of LN. This was similar to another Indian study by Panda SR et al. ${ }^{13}$ but the importance of this finding and its use for prognostication remains questionable as a rise in serum urea levels could be due to various reasons.

Microscopic haematuria, though was seen in class III LN, was most common in class IV LN. The probable reason could be that class IV LN is mainly a proliferative disease. ${ }^{24}$ In our study, the number of urine RBCs per high power field was found to increase across the classes with patients in class $\mathrm{V}$ LN having maximum RBCs in the urine. This is similar to many studies performed in other countries. ${ }^{10,25}$

Proteinuria is one of the important manifestations of $\mathrm{LN}^{26}$ as it has been consistently shown that those presenting with nephrotic range proteinuria have a worse outcome. ${ }^{25,27}$ Even in our study, we found that there was a statistically significant association between proteinuria and classes of $\mathrm{LN}$ with 24 - hour urine protein levels increasing as we go up the classes. We also found that patients with Class V LN had predominantly nephrotic range proteinuria with median proteinuria of $3151 \mathrm{mg} / 24$ - hour and this was consistent with previous studies. .2,28 $^{2}$

In our study, we found that C3 levels decreased with an increasing class of $\mathrm{LN}$ and the lowest $\mathrm{C} 3$ values were found in patients with class IV LN. This is in accordance with the results of another study done in Peru by Hurtado A et al.10 However, this was not true for $\mathrm{C} 4$ levels. One possible explanation for decreasing C3 levels with increasing class is that more than $50 \%$ of the glomeruli are involved in class IV and the glomeruli contain large amounts of immunoglobulin and complement in the mesangium and capillary walls. Also, there is going to be a higher activity with higher grade and hence more complement consumption and more antibodies against complements are expected. That being said, in our study, we found that C4 was low in both class III and class IV LN, while this particular study done in Peru ${ }^{10}$ showed class IV $\mathrm{LN}$ had the lowest $\mathrm{C} 4$ as well.

Even though our study failed to establish a significant correlation between anti - dsDNA titres and the classes of LN, it was observed that class IV had the maximum number of patients with ANA strong positive, anti - dsDNA titres $>800$ IU / $\mathrm{ml}$ and the highest mean of anti - dsDNA titres. This is in accordance with many studies published.9,10 The probable reason why we could not establish a significant correlation was due to the fewer number of patients in class $\mathrm{V}$.

Activity and chronicity indices proposed by the NIH tell if lesions are suggestive of active inflammatory injury ('activity' index) or irreversible scarring ('chronicity' index). Chronic, sclerotic lesions in glomeruli are thought to be irreversible and refractory to therapy, whereas active lesions are reversible and can respond to therapy. ${ }^{29}$ Several groups have reported that the activity index correlates with proteinuria, 
haematuria, and with renal function at the time of biopsy. Similarly, the chronicity index has also been reported to correlate with renal function. ${ }^{11,30}$ In our study, too, we found that activity score correlated significantly with the classes of LN with the highest activity score in class IV. However, a similar correlation could not be established for the chronicity index.

Overall, we found a significant correlation of symptoms, signs and laboratory parameters with the renal histological findings. However, owing to the cross-sectional nature of the study, lack of follow-up and no treatment correlation, we could not significantly determine the prognostic importance of each clinical and laboratory parameter on various classes of LN.

\section{CONCLUSIONS}

This study suggests some meaningful correlation between pedal oedema, facial puffiness, 24 hours urine protein, haemoglobin levels, C3 levels and activity score with lupus nephritis classes of ISN / RPS 2003 classification.

Even though based on this study, exact prognostication cannot be ascertained by clinical and lab parameters, it can be said that Class II LN patients had minimal symptoms, preserved GFR, low 24 - hour urinary excretion, low - normal complements and low levels of anti - dsDNA titres; Class IV, the most commonly encountered LN class, had maximum symptoms like pedal oedema and facial puffiness, high 24 hour urinary protein excretion (which may even be in the nephrotic range), very low complement levels (especially C3) and high levels of anti dsDNA titres and most of class $\mathrm{V}$ LN patients had nephrotic range proteinuria.

Data sharing statement provided by the authors is available with the full text of this article at jemds.com.

Financial or other competing interests: None.

Disclosure forms provided by the authors are available with the full text of this article at jemds.com.

The authors are grateful to the participants who provided the information for the study. The authors are grateful to Dr. Vasudeva Guddattu, Associate Professor, Department of Data Science, Manipal Academy of Higher Education for his help with the statistical analysis.

\section{REFERENCES}

[1] D'Cruz DP. Systemic lupus erythematosus. BMJ 2006;332(7546):890-4.

[2] Malaviya AN, Singh RR, Singh YN, et al. Prevalence of systemic lupus erythematosus in India. Lupus 1993;2(2):115-8

[3] Ruiz-Irastorza G, Khamashta MA, Castellino G, et al. Systemic lupus erythematosus. Lancet 2001;357(9261):1027-32.

[4] Ward MM, Pyun E, Studenski S. Mortality risks associated with specific clinical manifestations of systemic lupus erythematosus. Arch Intern Med 1996;156(12):1337-44.

[5] Cervera R, Khamashta MA, Font J, et al. Morbidity and mortality in systemic lupus erythematosus during a 10year period: a comparison of early and late manifestations in a cohort of 1,000 patients. Medicine (Baltimore) 2003;82(5):299-308.

[6] Satirapoj B, Wongchinsri J, Youngprang N, et al. Predictors of renal involvement in patients with systemic lupus erythematosus. Asian Pac J Allergy Immunol 2007;25(1):17-25.

[7] Christopher-Stine L, Siedner M, Lin J, et al. Renal biopsy in lupus patients with low levels of proteinuria. J Rheumatol 2007;34(2):332-5.

[8] Weening JJ, D'Agati VD, Schwartz MM, et al. The classification of glomerulonephritis in systemic lupus erythematosus revisited. J Am Soc Nephrol 2004;15(2):241-50.

[9] Satirapoj B, Tasanavipas P, Supasyndh O. Clinicopathological correlation in asian patients with biopsy-proven lupus nephritis. Int J Nephrol 2015;2015:857316.

[10] Hurtado A, Asato C, Escudero E, et al. Clinicopathologic correlations in lupus nephritis in Lima, Peru. Nephron 1999;83(4):323-30.

[11] Nasri H, Ahmadi A, Baradaran A, et al. Clinicopathological correlations in lupus nephritis; a single center experience. J Nephropathol 2014;3(3):11520.

[12] Nezhad ST, Sepaskhah R. Correlation of clinical and pathological findings in patients with lupus nephritis: a five-year experience in Iran. Saudi J Kidney Dis Transpol 2008;19(1):32-40.

[13] Panda SR, Kar CH, Sahu PK, et al. Clinico-pathological characteristics of lupus nephritis in eastern zone of india: a single tertiary center experience. Asian J Pharmaceutical and Clinical Research 2016:9(5):102-4.

[14] Satish S, Deka P, Shetty MS. A clinico-pathological study of lupus nephritis based on the International society of nephrology-renal pathology society 2003 classification system. J Lab Physicians 2017;9(3):149-55.

[15] Banfi G, Di Belgiojoso GB, Bosisio MB, et al. Morphological parameters in lupus nephritis: their relevance for classification and relationship with clinical and histological findings and outcome. Q J Med 1985;6(55):153-68.

[16] Magil AB, Puterman ML, Ballon HS, et al. Prognostic factors in diffuse proliferative lupus glomerulonephritis. Kidney Int 1988;34(4):511-7.

[17] Singh S, Zhou XJ, Ahn C, et al. A retrospective analysis of clinical presentation of lupus nephritis. Am J Med Sci 2011;342(6):467-73.

[18] Faurschou M, Dreyer L, Kamper AL, et al. Long-term mortality and renal outcome in a cohort of 100 patients with lupus nephritis. Arthritis Care Res (Hoboken) 2010;62(6):873-80.

[19] Halland AM, Bates WD, Tribe RD, et al. Lupus nephritis. Part ll. A clinicopathological correlation and study of outcome. S Afr Med J 1991;79(5):260-4.

[20] Mok CC. Understanding lupus nephritis: diagnosis, management and treatment options. Int J Womens Health 2012;4:213-22.

[21] Shobha V, Prakash R, Arvind P, et al. Histopathology of lupus nephritis: a single-center, cross-sectional study from Karnataka, India. International Journal of Rheumatology and Clinical Immunology 2014;2(1):OA3. 
[22] Austin HA 3rd, Boumpas DT, Vaughan EM, et al. Predicting renal outcomes in severe lupus nephritis: contributions of clinical and histologic data. Kidney Int 1994;45(2):544-50.

[23] Sircar D, Sircar G, Waikhom R, et al. Clinical features, epidemiology and short-term outcomes of proliferative lupus nephritis in Eastern India. Indian J Nephrol 2013;23(1):5-11.

[24] Markowitz GS, D'Agati VD. The ISN/RPS 2003 classification of lupus nephritis: an assessment at 3 years. Kidney Int 2007;71(6):491-5.

[25] Wallace DJ, Podell TE, Weinter JM, et al. Lupus nephritis. Experience with 230 patients in a private practice from 1950-1980. Am J Med 1982;72(2):209-20.

[26] Brugos B, Kiss E, Szodoray P, et al. Retrospective analysis of patients with lupus nephritis: data from a large clinical immunological center in Hungary. Scand J Immunol 2006;64(4):433-7.

[27] Neumann K, Wallace DJ, Azen C, et al. Lupus in the 1980s: III. Influence of clinical variables, biopsy and treatment on the outcome in 150 patients with lupus nephritis seen at a single center. Semin Arthritis Rheum 1995;25(1):47-55.

[28] Al-Zahrani IH, Qayyum A. Lupus nephritis. Clinicopathological correlation. Saudi Med J 2007;28(10):1503-5.

[29] David DJ, Schwartz MM. Lupus nephritis: histopathology. In: Madaio M, ed. Rheumatology and the Kidney. United Kingdom: Oxford University Press 2012:11-25.

[30] Mitjavila F, Pac V, Moga I, et al. Clinicopathological correlations and prognostic factors in lupus nephritis. Clin Exp Rheumatol 1997;15(6):625-31. 